\title{
Migration et pentecôtisme à Bruxelles
}

Expériences croisées

\section{Maïté Maskens}

\section{(2) OpenEdition}

Journals

Édition électronique

URL : http://journals.openedition.org/assr/16423

DOI : $10.4000 /$ assr. 16423

ISSN : $1777-5825$

Éditeur

Éditions de l'EHESS

Édition imprimée

Date de publication : 1 septembre 2008

Pagination : 49-68

ISBN : 978-2-7132-2191-0

ISSN : 0335-5985

Référence électronique

Maïté Maskens, "Migration et pentecôtisme à Bruxelles », Archives de sciences sociales des religions [En ligne], 143 | juillet-septembre 2008, mis en ligne le 01 juillet 2011, consulté le 30 avril 2019. URL http://journals.openedition.org/assr/16423; DOI : 10.4000/assr.16423 


\section{Maitté Maskens}

\section{Migration et pentecôtisme à Bruxelles Expériences croisées}

Depuis une trentaine d'années, des Églises pentecôtistes portées par des communautés immigrées ou issues de l'immigration ont fait leur apparition dans la capitale européenne. Elles connaissent aujourd'hui un essor non négligeable sur la scène religieuse belge. Leur implantation et leur succès grandissants vont de pair avec l'intensification des flux migratoires de ces trois dernières décennies en provenance d'Afrique sub-saharienne et d'Amérique Latine. Ces flux de migrations n’ont pas été organisés de manière systématique par les États concernés, l'année 1974 marquant l'arrêt officiel de l'immigration contingentée. La fermeture progressive des frontières externes du pays et le rétrécissement des filières légales de migration ont eu pour conséquence l'augmentation des migrations sous des formes illégales et clandestines. Les écrits sur ce sujet sont peu nombreux et disparates (Cornet, 2004 ; Kagné, Martiniello, 2001 ; Adam, Ben Mohammed, Kagné, Martiniello, Réa, 2002). Plusieurs fidèles sont en situation d'illégalité sur le territoire belge - ou l'ont été dans le passé - et la quasi-totalité d'entre eux ont vécu une expérience migratoire ou en sont les descendants directs. Ces problématiques occupent donc une place importante au cœur du dispositif religieux pentecôtiste.

L'articulation entre le pentecôtisme et le phénomène migratoire constitue le point de départ de notre réflexion. L'imbrication de ces deux phénomènes n'est pas caractéristique du pentecôtisme porté par des communautés d'immigrés en Europe puisque dans les pays du Sud, dont sont issus la plupart des fidèles, les Églises pentecôtistes implantées en villes sont souvent composées par des migrants d'origine villageoise (Robbins, 2004 : 123). Mais l'expérience de migration constitue un "moment privilégié pour la conversion" (Pédron-Colombani, 1998 : 149). Pourquoi ces Églises s’inscrivent-elles souvent dans un contexte de migration ? En quoi et comment le facteur religieux construit-il et donne-t-il du sens à l'expérience de l'immigration à Bruxelles ? Quelles sont les stratégies d'évangélisation en direction des " autochtones " du pays d'installation dans un contexte actuel d' "inversion " du courant missionnaire initié lors des premières vagues colonisatrices de l'Occident (Spindler et Bart-Lenoble, 2000) ? Quelles 
configurations particulières vont prendre ces mouvements religieux en pleine effervescence dans les pays du Sud (Corten, Mary, 2001 : 12 ; Corten, Dozon, Oro, 2003 : 17) dans un contexte religieux européen très différent, sachant que chaque groupe investit le pentecôtisme avec ses propres référents (Corten, MarshallFratani, 2001 : 11) ? Ces questions ouvertes constituent des formes d'interrogation particulières dans le paysage religieux contemporain et ce d'autant plus qu'elles sont susceptibles d'être appliquées à un grand nombre de pratiques religieuses en diaspora.

\section{Le paysage pentecôtiste bruxellois}

La composition des Églises pentecôtistes à Bruxelles est éclatée. Environ une centaine d'Églises pentecôtistes sont répertoriées dans l'annuaire du Conseil administratif du culte protestant-évangélique $(\mathrm{CACPE})^{1}$ pour la région de Bruxellescapitale. Néanmoins, beaucoup d'autres Églises existent en dehors de toute reconnaissance institutionnelle. Les raisons de cette invisibilité dans l'espace public sont multiples. La clandestinité de nombreux membres peut amener l'organisation à vivre dans l'ombre. Pour d'autres Églises, c'est l'aversion affichée pour une quelconque forme d' "alliance » avec l'État, considéré dans ce cas comme la personnification de Satan lui-même, qui entraîne une existence discrète. Une certaine méfiance s'est installée par rapport au monde extérieur (principalement par rapport aux journalistes, sociologues et autres enquêteurs curieux) depuis la fin des années quatre-vingt-dix suite à la logique de "persécution " engendrée par les polémiques autour de la rédaction du rapport parlementaire sur les sectes $^{2}$.

La diversité règne en matière de composition nationale des Églises et nous avons restreint le champ de cette étude ${ }^{3}$ à deux communautés de migrants, à savoir les fidèles originaires d'Amérique Latine et d'Afrique subsaharienne qui

1. Cette structure administrative de coopération fut créée en 2002, après de longues négociations à propos de l'éventuelle reconnaissance de nombreuses Églises protestantes à tendance évangélique. Ce conseil est désormais l'unique interlocuteur représentant les divers cultes protestants auprès des autorités publiques.

2. Rapport de la Chambre des Représentants de Belgique, «Enquête parlementaire visant à élaborer une politique en vue de lutter contre les pratiques illégales des sectes et le danger qu'elles représentent pour la société et pour les personnes, particulièrement les mineurs d'âge ", troisième session de la 49e législature, 28 avril 1997 (Duquesne, Willems, Partie II).

3. Cet article prend corps dans le cadre d'une thèse de doctorat entreprise en janvier 2006 grâce à une collaboration entre le Centre d'étude des religions et de la laïcité (CIERL) et le Centre d'anthropologie culturelle (CAC) de l'Université Libre de Bruxelles et provisoirement intitulée «Les Églises chrétiennes émergentes à Bruxelles. Recompositions identitaires et pratiques religieuses dans les assemblées pentecôtistes composées de migrants originaires d'Afrique subsaharienne et d'Amérique Latine sur le territoire bruxellois. » sous l'égide du Fonds national de la recherche collective (FRFC). Je remercie Pierre Petit, Jean-Philippe Schreiber, David Berliner et Joël Noret de m'avoir accorder du temps et de l'attention. 
constituent la grande majorité des assemblées pentecôtistes à Bruxelles. J'ai réalisé, au cours des années 2006-2007, plus d'une cinquantaine d'entretiens enregistrés auprès des membres de sept assemblées différentes, de nombreux entretiens informels (l'enregistreur devenant indécent au vu d'une intimité grandissante) et participé à de nombreuses activités religieuses de ces mêmes Églises ${ }^{4}$.

La taille de ces assemblées est variable et il est difficile d'en quantifier les fidèles tant les modes d'adhésion religieuse diffèrent en fonction de chaque individu. En effet, certains fidèles déclarent appartenir à une Église alors qu'ils ne la fréquentent plus ou très peu. Si l'on prend le critère de l'assistance au culte dominical, on dénombre plus de deux milliers de personnes pour la plus grande église pentecôtiste de la capitale mais seulement une vingtaine de fidèles environ pour celles de moindre importance numérique. La composition des Églises constituées majoritairement par des individus originaires de l'Afrique sub-saharienne à Bruxelles suit, dans la majorité des cas, des logiques de rassemblement sur une base régionale ou nationale. Les Congolais, ressortissants de l'ancienne colonie belge, constituent, pour des raisons historiques évidentes, le groupe le plus important, et l'on peut même parler d'Églises « kinoises » pour qualifier certaines Églises constituées presque exclusivement par des personnes originaires de la capitale congolaise. L'explosion et le succès des Églises de guérison dans ces mêmes régions (Devisch, 1998 ; 2001) expliquent la prédominance d'Églises congolaises sur la scène religieuse pentecôtiste bruxelloise, quand bien même ces pratiques émergentes à Bruxelles ne peuvent être réduites à une simple reproduction de celles qui ont cours au Congo-Kinshasa. L'apparente homogénéité culturelle de certaines Églises « congolaises » - au travers des rythmes musicaux, du lingala comme langue de louange, du pastorat d'origine congolaise - concourt à occulter la diversité culturelle condensée dans ces espaces religieux. Des fidèles originaires d'autres régions d'Afrique (Bénin, Cameroun, Burkina Faso, Niger, Burundi, Madagascar) sont en quelque sorte " congolisés » par leur appartenance religieuse. Ces assemblées ne peuvent donc être considérées ni comme de simples excroissances religieuses du Congo en Belgique, ni comme des constructions totalement nouvelles dans le pays d'immigration (Le Gall, 2003 ; Bava, 2003). Elles se situent dans un nouvel espace et conjuguent de manière subtile les liens affectifs ou imaginaires avec le(s) pays d'origine et les rapports souvent ambigus au pays d'accueil. En ce qui concerne les Églises composées par des hispanophones d'origine latino-américaine, on ne retrouve pas de groupe national prédominant sur la scène religieuse. C'est la pluralité d'appartenance nationale qui prévaut. Il est fréquent de rencontrer des fidèles aux origines géographiques diverses - Équatoriens, Péruviens, Colombiens, Guatémaltèques, etc. - sur les bancs d'une même Église. Ce pluralisme est favorisé par l'usage d'une langue

4. Je me suis investie à des degrés divers dans les Églises suivantes : la Nouvelle Jérusalem, l'Assemblée du Plein Évangile, la Cité Béthel, le Centro evangelico Emmanuel, l’Église internationale de Belgique, le Temple des Disciples de Jésus-Christ et l'Assemblée des Rachetés. 
commune et s'explique aussi par la plus faible densité et la plus grande diversité d'origine des immigrants latino-américains en Belgique.

La situation de ces deux "communautés ${ }^{5}$ n'est pas identique. J'ai cependant fait le choix méthodologique de m'attarder sur les ressemblances plutôt que sur les éléments qui séparent les groupes de fidèles ${ }^{6}$. La focalisation sur l'expérience de la migration est première dans mon approche. Dans les deux cas, ces associations religieuses illustrent l'hétérogénéité des mondes migratoires puisqu'elles rassemblent en leur sein des individus issus de vagues et de motifs migratoires très différents. Cependant, cette sensibilité pentecôtiste partagée par les deux groupes semble fonctionner comme un élément fédérateur dans ce contexte particulier. La présence d'une minorité d'individus originaires d'Amérique Latine dans des Églises composées en grand nombre par des Africains et inversement met en lumière une propension à la collaboration sur la base d'affinités religieuses entre ces personnes originaires de continents différents mais, avant tout, marquées par une expérience migratoire et un statut généralement dévalué dans la société d'accueil. Pour certains interlocuteurs, leur intégration dans l'Église d'une communauté de migrants différente de leur groupe d'origine semble l'opportunité d'échapper à la pression sociale des autres « frères et sœurs en Christ ". C'est le cas d'une interlocutrice guatémaltèque qui a changé d'Église après son divorce pour se retrouver dans une assemblée pentecôtiste à majorité africaine. Elle explique qu'elle se sent bien dans cette "ambiance » jusque-là inconnue. D'autre part, les autorités de son Église précédente lui avaient demandé de quitter ses fonctions à l'école du dimanche après avoir pris connaissance de sa décision de rompre ses liens matrimoniaux. Ce rapprochement se manifeste aussi au travers de divers évènements « latino-africains » organisés en tandem par certaines Églises autour d'activités cultuelles partagées. Ces rencontres se donnent pour titre "réseau prophétique " ou encore "alliance pour Jésus » et illustrent la tendance à la création de mondes en réseau qui caractérisent le pentecôtisme dès son origine. Dans ce genre de rassemblement, ce n'est pas l'identité ethnique qui est retenue comme support fédérateur mais bien leur appartenance chrétienne (Glick Schiller, Çaglar, Guldbrandsen, 2006 : 615). Les fidèles d'origines différentes se reconnaissent et se définissent avant tout comme des « frères et sœurs en Christ ».

5. L'emploi de ce concept doit être nuancé car il suppose une certaine homogénéité qui n'existe pas forcément dans la réalité empirique. Voir à ce sujet les écrits de Rogers Brubaker et Frederick Cooper (2000) qui critiquent l'usage de concepts tels que « communauté ", "classe ", " race » et surtout celui d' « identité » qui, sous leurs évidentes apparences, cachent des processus sociaux plus complexes.

6. Déjà dans les pays d'origine des fidèles, la composition sociale des assemblées suit des logiques radicalement différentes. Alors qu'en Amérique Latine, le pentecôtisme touche avant tout les groupes les plus paupérisés, dans les villes africaines ce sont principalement les classes moyennes qui constituent les assemblées (Corten, Marshall-Fratani, $2001: 20$ ). 
Cependant, dans ces Églises marquées par des ambitions missionnaires d'intensité différente en direction des Belges, on retrouve très peu de personnes d'origine européenne, de " gringos ", de " blancs ", de " caucasiens " ou encore de "gaulois » pour reprendre des expressions fréquemment formulées par les interlocuteurs. Quand il y en a, ceux-ci constituent invariablement une très petite minorité et font d'emblée la fierté de l'Église. L'attention dont je fais l'objet lors des cultes et les nombreuses photos où j'apparais (parfois sur le site Internet des certaines Églises) en témoignent ${ }^{7}$. La tension est alors de mise dans ces Églises oscillant entre un discours d'ouverture - dicté par l'idéal d'universalité du message chrétien " Jésus est venu pour tout le monde » et donc la nécessité de représenter tous les mondes sociaux en son sein - et la composition réelle des Églises qui continuent d'être des lieux d'entre soi et parfois même des « ghettos » comme les qualifient certains fidèles eux-mêmes. Ceci nous amène à examiner de plus près l'idée selon laquelle l'adhésion à ces Églises prend en grande partie son sens dans un contexte de migration.

\section{Le " Dieu des immigrés"}

Le thème de la migration est un sujet omniprésent, objet de nombreux discours des gens d'Églises. Bien souvent, la présence des fidèles sur le sol belge est, d'abord, comprise, ou tout au moins expliquée, comme la matérialisation d'une volonté divine. C'est Dieu qui a choisi de les «mettre " dans ce pays, de les déplacer ou de déplacer leurs parents. Cette interprétation rétrospective couvre une multitude d'itinéraires religieux et migratoires différents. Mais, dans la plupart des cas, deux figures ressortent de manière récurrente. Le profil religieux le plus classique est celui d'un ancien fidèle catholique touché par ce "protestantisme émotionnel» (Willaime, 1999: 5) dans son pays d'origine et qui, dès son arrivée en Belgique, se met en quête d'une Église répondant à ses attentes. Dans d'autres cas, plus rares, ce sont les réseaux religieux qui font se déplacer les pasteurs ou les étudiants en théologie les plus méritants. La religion est alors génératrice de mobilité (Le Gall, 2003 ; Bava, 2003 ; Meinteil, LeBlanc, 2003 ; Noret, 2005).

Dans le contexte bruxellois, la mise sur pied de ces Églises répond souvent à l'absence de structures religieuses satisfaisantes pour les nouveaux arrivants en Belgique. Ceux-ci racontent être étonnés de trouver la porte des Églises locales fermées à certaines heures, les lieux de culte vides et décrivent les modes d'expression religieuse occidentaux comme « froids » ou trop rigides. C'est principalement la religion catholique et son déclin qui sont visés ici. La volonté de

7. Vu l'économie de ce texte, je ne m'attarderai pas dans ce travail sur les dimensions méthodologiques, les subtilités et les tensions de mon intégration dans les différentes assemblées. Plus de détails sur le thème délicat de l'implication du chercheur dans des Églises pentecôtistes sont développés dans les articles de Ruy Llera Blanes (2006), de Sandra Fancello (2006) et de Géraldine Mossière (2007). 
se regrouper pour prier dans leur langue d'origine semble déterminante dans de nombreux cas. Ces Églises prennent alors souvent la forme de clubs de prière durant leurs premières années d'existence. On se réunit chez l'un ou chez l'autre fort investi dans une assemblée de son pays d'origine - le pentecôtisme touche environ $10 \%$ de la population en Amérique Latine et en Afrique (Corten, 2004 : 1) - qui reproduisent alors en Europe ce qu'ils ont connu ailleurs. D'autres assemblées émergent de la volonté d'un fidèle particulier de fonder sa propre Église après avoir fréquenté une assemblée de ce genre en Belgique. Dans d'autres cas de figure, la création de ces Églises correspond à des stratégies missionnaires préétablies en provenance de grandes dénominations pentecôtistes transnationalisées visant les populations immigrées d'Europe occidentale. C'est le cas de la Nouvelle Jérusalem, Église pentecôtiste de taille importante (environ trois mille fidèles dans toute la Belgique) dont la création à la fin des années septante correspondait à la volonté d'un missionnaire nord-américain d'origine mexicaine de la Church of God de rassembler les "Africains » de Bruxelles dans une Église (Maskens, Noret, 2007).

On peut observer une évolution dans ce domaine puisque, bien des années plus tard, ce n'est plus le désir d'être entre soi qui est valorisé dans la grande majorité des Églises mais bien l'universalité du message évangélique et l'ouverture des portes de l'Église à toutes les personnes «sans distinction de race, de couleur, ni de catégorie sociale » pour reprendre les mots d'un pasteur péruvien. Suivant cette logique, un effort de traduction des cultes en plusieurs langues, ou tout au moins en français ou en néerlandais, est fait de façon systématique dans les grandes Églises et de manière plus informelle dans les Églises de moindre importance numérique (qui répondent alors plutôt de manière spontanée aux langues parlées par les fidèles présents au moment du culte). Cette volonté d'ouverture affichée des Églises se traduit aussi par des changements d'appellation : des assemblées portant des noms faisant référence au continent africain ou latinoaméricain, à l'un des pays de ces continents ou encore des noms en langues étrangères se transforment pour laisser place à des qualificatifs tels que "Église internationale » ou encore " universelle » qui soulignent davantage la continuité avec le message chrétien de portée universelle. Les références culturo-nationales sont peu à peu gommées pour laisser place à une identité religieuse transnationale.

Dans la vie quotidienne des Églises, la préoccupation liée au phénomène migratoire et aux difficultés d'insertion dans la société d'accueil est permanente. Monique Misenga décrit les « couches sociales » représentées dans deux groupes religieux africains, en Wallonie et à Bruxelles en 2000, dans lesquels les migrants en situation délicate constituent la plus grande partie des fidèles. Elle écrit au sujet du groupe Nzambe Malamu : "la plupart des membres nous ont semblé (être) des réfugiés politiques, d'autres avaient des problèmes de papiers. Plusieurs d'entre eux faisaient leurs démarches soit pour devenir Belges, soit pour régulariser la situation (...) la communauté priait pour eux »(Misenga, 2000 : 246). Abel Ugba décrit la situation similaire des migrants africains à Dublin où les 
activités pentecôtistes " are uniquely relevant to the situations of Africans immigrants (...) very often themes in prayers, songs and sermons point to God as the direct and sure answers to social and political problems, including racism and immigration difficulties »(Ugba, 2006 : 169). À Bruxelles, on retrouve ces préoccupations de régularisation et de légitimation au premier plan parmi d'autres sujets de prières récurrents : trouver un emploi, trouver un conjoint, assurer sa santé et la stabilité de sa famille. Les réponses apportées par les Églises à ces demandes se structurent autour de deux niveaux d'action : le niveau concret et celui de l'action divine. Le premier niveau fait apparaître ces Églises comme des structures d'aide aux migrants nouvellement arrivés, épaulés par les fidèles établis en Belgique ayant déjà rencontré les mêmes difficultés. Il n’est pas rare alors qu'une partie des lieux de culte soit consacrée à l'hébergement des personnes en situation illégale. Certaines Églises mettent à disposition des fidèles des avocats et des juristes africains offrant gratuitement leurs services et leur aide dans la constitution et le suivi des dossiers relatifs à la régularisation du statut de migrant. D'autres marques de solidarité se manifestent par l'hébergement d'un "frère " par un autre mieux installé, par le partage fréquent de repas après certains cultes, ou encore par l'achat d'un abonnement de transport en commun grâce aux dons des fidèles. Ces pratiques de soutien permettent aux personnes en situation délicate de survivre un certain temps dans un nouvel environnement plutôt hostile. En d'autres termes, ces " enfants de Dieu " se serrent les coudes au sein d'un réseau social dense et émotionnellement coloré. C'est ce tissu social émergeant au niveau local qui assure aux migrants l'aide la plus efficace pour autant que le nouveau venu témoigne de sa bonne foi et apporte les preuves de sa ferveur religieuse. En effet, la contrepartie implicite attendue de la personne qui bénéficie de ce soutien est de montrer qu'il se repose entièrement sur Dieu, qu'il s'abandonne aux actions de l'Esprit Saint et qu'il prie sans relâche pour voir sa condition s'améliorer. Cette configuration particulière entraîne une hétérogénéité des catégories socioéconomiques dans l'Église. Une minorité de fidèles ayant " réussi » (en situation régulière en Belgique, détenteurs d'emploi plus ou moins prestigieux et présentant un modèle familial hétérosexuel monogame de stabilité) servent d'exemples aux fidèles dans des situations précaires (cherchant à régulariser leur situation, à trouver l'âme sœur, à sortir de l'indigence ou à faire face à des problèmes de santé).

Au niveau de l'action divine, les fidèles dessinent les contours d'un Dieu particulier, celui des « réfugiés ». Certains pasteurs racontent comment cultiver la foi en ce "Dieu des réfugiés" qui a déjà réalisé bon nombre de miracles comme le décrit Louis ${ }^{8}$, pasteur centrafricain :

8. Les noms des pasteurs et des fidèles ont été modifiés en vue de préserver leur anonymat. Les détails concernant leurs Églises ne sont pas exposés, dans le même souci de discrétion. En effet, le débat passionné de la fin des années quatre-vingt-dix en Belgique autour des dérives sectaires de certains mouvements religieux a marqué ces Églises et continue de définir, dans une certaine mesure, leur rapport souvent méfiant au monde extérieur. Tous les entretiens dont les extraits apparaissent dans cet article ont été réalisés au cours de l'année 2006 à Bruxelles. 
«Il y a beaucoup de témoignages où l'on peut voir comment Dieu à fait des merveilles dans la vie de beaucoup. À ceux qui ont reçu l'ordre de quitter le pays, expulsés et qui aujourd'hui sont Belges. Ils ont acheté des maisons, se sont mariés et ils ont vu que Dieu est vrai, Dieu existe, Dieu est vivant. »

Il faut donc refuser ces " habits" de victimes pour enfiler ceux de guerrier victorieux et «conquérant » du monde moderne puisque, comme dans l'Église Universelle du Royaume de Dieu, d'origine brésilienne, implantée dans plusieurs parties du monde, "l'image du perdant est constamment dépréciée " (Corten, Dozon, Oro, 2003: 29). Ce genre de discours contient un pouvoir de réenchantement du quotidien des immigrés, dont l'identité est constamment mise en doute, qui fait la force du pentecôtisme dans le contexte bruxellois. La puissance de ce "Dieu des réfugiés " s'étend jusque dans la sphère politique et ce sont alors des décisions en matière d'immigration qui sont comprises comme des miracles divins, réponses aux multiples prières humaines. Gerrie Ter Haar décrit cette reconstruction religieuse de l'action politique à l'œuvre dans les communautés chrétiennes ghanéennes d'Amsterdam qui voient la main de Dieu dans les changements de politique d'immigration (1998: 45). En Belgique, la campagne de régularisation ouverte au mois de janvier 2000, suite à l'entrée en vigueur de la loi relative à la régularisation de séjour de certaines catégories d'étrangers séjournant en Belgique, constitua pour beaucoup de chrétiens pentecôtistes une preuve tangible de la puissance de Dieu. De fait, plusieurs pasteurs font une lecture rétrospective de cet évènement et racontent aujourd'hui les sessions spéciales de prières intensives entreprises dans les mois précédant la décision. Ils disent leur ferme intention de l'époque de changer cette situation décrite comme « insoutenable » pour de nombreuses personnes en marge de la légalité.

Nombreux sont les évènements qui sont lus comme une réponse divine aux prières humaines. Ces réponses peuvent prendre une multiplicité de formes. Par exemple, un mariage "blanc" peut être synonyme de signe divin, comme pour ce fidèle colombien qui explique : "Nous, on prie et Dieu exauce... et si c'est en mettant un homme belge sur le chemin d'une jeune fille en recherche de régler ces problèmes, pourquoi pas? " Les manières divines de répondre aux prières humaines sont imprévisibles et dans ce domaine, seule l'expérience semble importer. Dans leur discours, c'est la continuité explicative qui est de mise entre deux registres d'efficacité différents. Les fidèles passent sans transition d'un registre à l'autre. Et l'on observe fréquemment un glissement explicatif entre cette activité divine, ce Dieu agissant au nom duquel on se mobilise et le réseau social qui est créé autour de lui et qui, pour sa part, répond concrètement aux attentes et aux demandes des fidèles. À cet égard, une comparaison avec le retour à l'islam de certains commerçants marocains de la région bruxelloise peut être éclairante. Albert Bastenier montre comment cette catégorie d'immigrés n'a redécouvert la religion que tardivement dans leur pays d'accueil. Ceux-ci racontent avoir appris seulement " par l'expérience de l'immigration que la religion est un lien puissant 
entre les hommes, sécurisant et inspirant la confiance mutuelle " (Bastenier, 1998 : 206). C'est bien le facteur religieux dans sa dimension fonctionnelle et utilitaire, qui est mise en relief ici. Grâce à leur ancrage religieux, ces commerçants acquièrent une réputation de confiance qui assure, en grande partie, le succès de leur négoce. Dans ce cadre, pour les pentecôtistes comme pour les musulmans de Bruxelles, c'est bien l'adhésion à un groupe religieux et ses effets de réseau qui assurent en partie une certaine insertion, et parfois même la réussite sociale.

\section{Ôter la « chape de béton invisible » : entre dépréciation et revalorisation}

Le message diffusé dans plusieurs Églises nous renseigne sur la situation parfois difficile des migrants. Plusieurs pasteurs dénoncent la stigmatisation dont leurs membres sont victimes et un pasteur burkinabé m'explique que lors de la création d'une de ces Églises, dans les années quatre-vingt, ils se réunissaient pour prier et ainsi : " enlever la chape de béton invisible qu'il y a au-dessus de notre population, pour que vraiment nos gens qui sont en Belgique puissent vraiment respirer, vivre, être normal, qu'on soit normal dans le pays. » Comment ne pas voir dans cette évocation métaphorique la dépréciation sociale qui touche ces nouveaux venus en Belgique? « Être normal » dans la société belge reviendrait alors à ne plus être renvoyé, de manière récurrente, à une forme de dépréciation identitaire et d'assignation permanente à l'altérité.

Dans les premiers temps de leur arrivée en Belgique, les fidèles immigrés ou descendants d'immigrés racontent leurs tentatives de répondre à l'injonction normative "d'intégration ", sorte de compensation implicite à leur présence sur un territoire étranger. "Être intégré » reviendrait alors à ressembler le plus possible aux Belges, à se fondre dans la masse. Aujourd'hui, malgré de nombreux efforts, cette possibilité d'intégration véritable dans la société semble être mise en doute. Ils constatent, avec amertume, que leur bonne volonté et leur désir d'adaptation ne semblent pas suffisants pour résoudre l'asymétrie des rapports sociaux. Ce refus d'acceptation et de reconnaissance de la société d'établissement se traduit concrètement, selon les interlocuteurs, par les emplois subalternes qui leur sont généralement octroyés. C'est d'ailleurs souvent à ce moment du discours que les interlocuteurs saisissent l'occasion de dire qui ils étaient dans leur pays d'origine et de souligner la dépréciation de statut qu'ils ont subie lors de ce déplacement. Un jeune fidèle originaire de République démocratique du Congo rapporte la condition dans laquelle se retrouvent les "parents " immigrés congolais en Belgique malgré leurs brillants parcours universitaires :

«Et quand ces enfants voient leurs parents terminer leurs études pour lesquelles ils étaient venus ici et regardent la lutte pour la survie... Ces enfants n'ont plus d'attache dans leur pays d'origine et les parents sont obligés de vivre ici parce que leurs enfants étudient et sont nés ici. On doit leur assurer un cadre de vie qui soit meilleur pour 
eux pendant qu'au pays, ça ne va pas. On ne peut pas les ramener au pays dont on sait qu'il n'y a plus d'espoir, surtout pour la jeunesse. Et les parents font comme tout les autres, ils restent à s'intégrer. Et malheureusement, à cause de leurs diplômes, qui pour la plupart, sont des grands diplômes, ils n'arrivent pas à trouver du travail et ils restent à la maison à zapper. Les enfants qui vont à l'école, quand ils rentrent, ils trouvent leurs parents devant l'écran de la télé, ils zappent, ils zappent. Ils se disent : "bon, papa là, il a fini ses études, mais pourquoi il ne travaille pas ?" Et le Papa leur répond: "mais je ne sais pas travailler parce qu'on me donne pas de travail à cause des études, à cause de mon diplôme". Oui parce que pour la plupart, ce sont des docteurs, ils ont de trop grands diplômes. "

Les fidèles regrettent donc de ne pouvoir jouir des mêmes avantages et disent vivre selon un régime autre où la différence est retenue comme critère de discrimination. C'est un fait avéré dans les sociétés "multiculturelles " contemporaines que toutes les cultures ne sont pas inscrites de la même manière dans le paysage socioculturel (Réa, 1998). Certaines cultures semblent plus «valables» que d'autres et en dernier recours, c'est la conformité avec le modèle culturel dominant qui fonctionne comme critère ultime. C'est donc bien un "déficit de légitimité » qui continue d'entourer le «statut » des immigrés et de leurs descendants (Réa, Tripier, 2003 : 7). Un ensemble de logiques sociales et de mécanismes complexes concourent à les maintenir dans cette position de «citoyens de seconde zone» dans la Belgique postcoloniale ${ }^{9}$.

Le message religieux des Églises pentecôtistes tente de répondre à cette impasse en offrant un cadre interprétatif à la condition des fidèles et en donnant du sens à leur inscription sur ce territoire. Le discours religieux est, en outre, articulé de telle façon qu'il peut catalyser les angoisses, les frustrations ou encore les humiliations, fruits d'une situation sociale asymétrique. Il entre de ce fait en résonance avec le ressenti et l'insatisfaction accumulés dans certains cas par les migrants. Ce message est l'opportunité d'une mise en récit alternative du fait migratoire. Les métaphores bibliques sont d'usage pour donner du sens à cette situation et encourager de la sorte les fidèles à sortir de la "misère ". À cet égard, le pasteur congolais Nestor compare ses fidèles au peuple d'Israël en exil hors d'Égypte :

«Quand Dieu a sorti Israël de l'Égypte, il ne les a pas envoyés pour mourir. Il les a envoyés comme le dit la Bible où coulent le lait et le miel. Et quand ils sont arrivés, quand tu entends Dieu parler, tu as l'impression que tu t'asseois dans ton salon, tu ouvres la bouche et le miel coule dans ta bouche ? Non, ce n'est pas comme ça, ils ont dû travailler pour avoir ça (...) Le lait et le miel ne vont pas venir simplement parce qu'on est en Belgique!"

Le lait et le miel représentent les possibilités économiques du pays d'accueil. Pour en bénéficier, ce sont les valeurs du travail et de l'effort qui sont mises en avant. On incite les jeunes et les moins jeunes à suivre des formations, à se

9. Il existe une certaine continuité entre les représentations contemporaines de l'immigré et l'imaginaire colonial du siècle dernier en Occident (Blancel, Blanchard, 1997). 
«mettre dans les structures » de la société d'accueil et à prendre part activement à la vie de celle-ci. La victimisation n'est pas de mise. La figure valorisée du pentecôtiste contemporain est assez éloignée de celle du chrétien cultivant la "pauvreté » de la tradition catholique. La loi de la prospérité est un motif théologique primordial dans ces mouvements religieux (Gifford, 2001). Pour les interlocuteurs pentecôtistes, la réussite sociale est l'une des voies du salut et l'indigence ne fait pas partie du "plan de dieu ». Il convient donc de porter sur soi les signes des multiples bénédictions divines. Un rapide coup d'œil à la sortie d'un culte du dimanche le confirme. Les tenues vestimentaires des fidèles et les voitures de luxe qui défilent sont autant de manifestations de cette valorisation de la richesse ${ }^{10}$. Ces signes constituent les preuves matérielles d'un Dieu vivant et agissant et, par la même occasion, donnent du poids à la ferveur religieuse du fidèle qui a réussi.

De manière concrète, pour faire face à cette "chape de béton invisible ", les autorités pastorales encouragent les fidèles à une mobilité sociale ascendante dans leur vie mondaine. Il est vrai que le pentecôtisme a la particularité, comme beaucoup d'autres mouvements religieux ${ }^{11}$, de porter son attention principale sur le présent immédiat. Il s'agit bien d'une culture religieuse de l'instant puisque l'on se préoccupe du salut des fidèles hic et nunc (Willaime, 1999). Ce sont les problèmes concrets du quotidien et leur résolution qui sont visés lors des cultes et autres activités religieuses.

D'autres passages bibliques sont retenus pour donner du sens à la condition de migrants, la Bible étant la seule source de vérité légitime dans le monde protestant (Willaime, 1992). Trois versets viennent de manière récurrente dans les propos des pasteurs et lors de discussions entre fidèles. Il s'agit de ceux qui font référence à la notion de refuge et d'inscription d'un peuple sur un territoire : «Heureux tous ceux qui se réfugient en l'éternel. "; " Heureux tous ceux dont il est le refuge. »(Ps. 2, 12), "Celui qui se réfugie en lui héritera du pays. »; "Mais qui se réfugie en moi aura pour lot la terre et pour héritage ma montagne sainte. » (Is. 57, 13) et encore : "Tous lieux que foulera la plante de ton pied, je te le donne. "; "Tout lieux que foulera la plante de vos pieds, je vous l'ai donné, comme je l'ai dit à Moïse. » (Jos. 1, 3). Ces versets concourent à donner une base biblique à leur inscription sur ce territoire. Ils sont retenus car ils donnent un regard nouveau sur ce qui est souvent identifié par les Belges comme une présence illégitime au sein de leur nation que les migrants n'ont pas contribué à «constituer ». Les migrants les plus stigmatisés étant souvent décrits par les tenants d'une approche réductrice du phénomène migratoire comme un « fléau "

10. Cette description est valable pour les Églises composées de personnes originaires d'Afrique subsaharienne, il n'en va pas de même pour les Églises composées de fidèles latinoaméricains.

11. On pense ici aux « nouveaux mouvements religieux » de type New Age dont l'attention est également portée sur le bien-être au quotidien (Hervieu-Léger, Davie, 1996). 
en train d'envahir le continent européen (Martiniello, 2001 : 5). En ce sens, la Bible offre un modèle d'inclusion alors que la tendance actuelle de la société occidentale reposerait plutôt sur un modèle d'exclusion des étrangers (Ter Haar, 1998 : 47). L'interprétation de certains passages bibliques permet aux fidèles d'articuler leurs sentiments d'injustice, de souligner la vanité du monde des hommes, et, enfin, de critiquer l'inégalité et l'asymétrie des rapports sociaux en cours dans la société d'accueil.

\section{La mission en retour : un rôle pour les fidèles migrants ?}

Revêtir «l'habit religieux » offre l'opportunité aux migrants de changer de statut. De nombreux pentecôtistes se présentent comme les "sauveurs" d'une Europe de plus en plus « décadente ». Dans ce cadre, le devoir chrétien d'évangélisation est pris très au sérieux. Les fidèles africains subsahariens donnent à ce devoir "d'essaimer " le nom de "mission réciproquée » ou encore de " mission en retour " en référence à ce mouvement inverse et inattendu de diffusion du message chrétien de l'Afrique post-coloniale vers l'Europe. Par une boucle de l'histoire, ces nouveaux christianismes reviennent au point d'origine grâce aux migrations de chrétiens du Sud initialement évangélisés par les chrétiens occidentaux (Spindler, $2000: 22$ ). Aujourd'hui l'espace européen post-colonial est caractérisé, selon eux, par une diminution inquiétante de la ferveur religieuse. Ferveur qui pourtant, selon leurs dires, aurait grandement contribué à la prospérité de ces mêmes régions. La conséquence de cette réduction de la dimension religieuse dans la vie quotidienne des Européens est perçue comme une déchéance morale. Ce désintérêt religieux entraîne une "perte de repères " laissant libre cours à de nombreux comportements «immoraux ". Le " désordre » actuel qui en découle est observable selon les interlocuteurs au travers de l'augmentation du nombre de divorces, de la légalisation du mariage entre personnes de même sexe ou encore de la "débauche " sexuelle à laquelle s'adonnent les jeunes européens. Ces « dégradations " de la morale sont retenues comme autant de preuves tangibles de la disparition du Dieu chrétien dans le «cœur» des Européens. La situation est semblable dans les Églises composées majoritairement de sudAméricains qui partagent ce jugement sur les Européens, auxquels ils n'aspirent d'ailleurs pas à ressembler. C'est pourquoi la préservation de l'identité culturelle et religieuse peut prendre des allures de voie de salut.

Le prosélytisme traditionnel tente de répondre à cette carence de moralité suivant des logiques de propagation qui lui sont propres. On demande aux fidèles, qui deviennent tous des évangélisateurs potentiels, de ramener des membres de leur famille, des amis, ou encore des collègues et, pourquoi pas, des Belges non immigrés (on non issus de l'immigration) au culte. D'autre part, des pratiques spécifiques plus ciblées ont cours également dans le cadre de cette mission de "régénération morale " (Fancello, 2005). Il s'agit par exemple de soirées d'évangélisation destinées à sauver les "prostituées et les homosexuels " 
de la ville. Après un culte pour appeler la protection, la puissance et la force divine, les fidèles "s'arment» de plusieurs bibles et de thermos de café pour arpenter les quartiers interlopes de Bruxelles afin de déposer une "graine" de foi chrétienne dans le cœur des prostituées. Ces " gens de mauvaise vie » représentent la cible idéale pour ces «nouveaux chrétiens ». Il est intéressant de souligner que, même dans ce cadre, les pentecôtistes se tournent de manière préférentielle, pour des raisons de facilité communicationnelle, vers les ressortissants de leur région d'origine. C'est auprès d'eux qu'ils insisteront le plus fermement.

Cette volonté de ramener les « brebis égarées » s'exprime aussi au travers du discours d'ouverture et du rôle dévolu à l'Église. De nombreux pasteurs expliquent que s'ils sont dans ce pays, ce n'est pas pour rester entre eux et ainsi contribuer à former un ghetto ethno-national mais bien pour amener les autochtones à franchir les portes de l'Église. Ils soulignent l'absurdité d'une Église composée par des individus de même nationalité et Lucien, pasteur originaire de République Démocratique du Congo affirme à cet égard que :

"C'est Dieu qui a voulu qu'on soit en Belgique, qui nous a retirés du Congo et qui nous a établis ici en tant que ministre de culte. C'est pourquoi on a le devoir d'aller vers les Belges pour ne pas bâtir une île de Congolais, une Église rien que pour les Congolais, parce que ça, on pouvait rester au Congo pour faire ça. »

Le cas congolais est spécifique car les ressortissants de ce pays d'origine ont pour la plupart été éduqués dans des écoles catholiques par des pères blancs d'origine belge. Ils racontent d'ailleurs souvent leur étonnement par rapport au «néant spirituel » qu'ils perçoivent en Belgique. À l'image des missionnaires de leur enfance investis d'une foi conquérante, ils s'attendaient à trouver un pays peuplé de fervents chrétiens. Cet extrait met en lumière la nécessité éprouvée par ces migrants d'aller vers la population belge non immigrée pour lui apporter la bonne parole. Ils souhaitent se présenter comme une valeur ajoutée pour la société d'accueil et, dans le même mouvement, inscrire leur présence sur le territoire de manière positive. C'est en quelque sorte un moyen pour combler ce manque de légitimité qui caractérise leur position dans ce nouvel espace, une manière de rétablir leur respectabilité sociale.

Grâce à ce discours, ils substituent le statut de chrétien engagé à celui qui leur est souvent associé de " profiteurs ». De « voleurs de travail », ils deviennent des prophètes des temps modernes. On peut donc comprendre une partie de ce phénomène comme une stratégie répondant à la dépréciation générale qui les entoure en vue de mieux se positionner ou même, pourquoi pas, de renverser la hiérarchie sociale en cours. Cette substitution peut être comprise comme un processus de réappropriation de leur identité, une manière de refuser l'assignation à la différence dont ils sont l'objet, de se positionner plus avantageusement dans le champ concurrentiel de groupes sociaux au sein de sociétés dites « multiculturelles ». Les «identités» ethniques ou culturelles n'existent pas en soi mais bien dans leurs rapports avec les autres « différents ». Cette différence peut alors 
prendre des contenus tout relatifs. C'est plutôt la construction et la permanence des frontières entre les groupes qu'il importe de questionner (Barth, 1969). Il en va de même en ce qui concerne la définition de certaines expressions religieuses controversées qui n'existent jamais dans l'absolu mais bien dans un face-à-face avec ceux qui les dénoncent (Luca, 2007).

Cependant, la «mission en retour » semble être un échec et les Européens ne sont pas nombreux lors des cultes. Ces Églises restent en grande partie des lieux d'entre soi où l'on parle une langue vernaculaire et où l'on se rassemble selon les modes de sociabilité des pays d'origine. Les références culturelles lors des cultes sont constantes: qu'il s'agisse de la nourriture, des styles musicaux, de certaines danses ou encore de certains modèles d'autorité. Les fidèles s'accordent alors pour constater que cette "ethnicité » affirmée peut mettre les Européens «mal à l'aise ». Un jeune belgo-congolais déclare à ce propos : «tu sais, les Blancs que tu vas rencontrer dans l'Église, ce seront vraiment des négrophiles ». Il sous-entend que la minorité d'Européens qui se rend au culte nourrit forcément une certaine fascination pour l'Afrique et que cette fascination culturelle est première et décisive en regard de l'adhésion strictement religieuse de ces Européens. Plusieurs traits spécifiques de la population belge non immigrée sont mis en avant pour expliquer l'échec de l'attraction de ceux-ci dans les Églises. On dit de ces derniers qu'ils passent mais ne s' "attachent " pas et se caractérisent par un manque de persévérance vis-à-vis de la longue durée des cultes, une certaine rigidité face à des horaires fluctuants, une incompréhension des modes d'expression de la foi pentecôtiste, et surtout une autosuffisance économique, sorte de "blocage » ultime qui les empêchent de s'approcher réellement de Dieu. En dernier recours, c'est le racisme des Européens qui est l'argument final. Le constat de cette incompatibilité religieuse semble peiner les fidèles et souvent l'amertume colore le bilan de ce revers missionnaire. Malgré le rejet et l'indifférence qu'inspire le plus souvent ce christianisme controversé vécu par des populations marginalisées, les fidèles continuent d'avancer les arguments d'universalité dont ils sont porteurs, comme si cela constituait la seule condition de leur valorisation.

Certaines autorités religieuses protestantes plus anciennes ne reconnaissent pas aux Églises pentecôtistes à forte densité de migrants leur prétention à l'universalité. En effet, certains responsables tendent à désigner davantage ces Églises au travers du prisme ethnique qui semble plus acceptable que celui de leur dimension religieuse. Pour d'autres, ces expressions de la foi ne sont que de pures aberrations religieuses. C'est ainsi que l'on peut lire dans un ouvrage du porteparole de l'Église protestante unie de Belgique que ces fidèles «s'expriment par des prières infantiles » lors de cultes qui sont de "véritables séances d'aérobics religieux » où "à l'exemple des communautés ethniques - ou la chose est aussi naturelle que culturelle - il semble que certaines assemblées ne peuvent plus entonner un hymne chrétien sans lever les bras, claquer des mains ou danser sur 
place» (Dandoy, 2005 : 155-156). Ce discours disqualifiant est relayé dans les médias qui continuent de définir ces Églises par l'appartenance nationale de la majorité de leurs membres. En d'autres termes, elles sont associées à leur " africanité " ou à leur "latinité " plutôt qu'à leur appartenance religieuse au grand groupe des religions chrétiennes. Je rejoins les propos de Gerrie Ter Haar au sujet des chrétiens ghanéens émigrés aux Pays-Bas quand elle avance que ceux-ci défient la chrétienté occidentale sur son propre territoire (1998: 8). Ce défi ne se pose pas tant en termes d'effectifs mais plutôt en termes de place assignée au christianisme dans un monde européen sécularisé. En effet, le projet de société proposé par ces Églises ne suit pas la délimitation opérée entre la sphère qui relèverait du privé et la sphère communément appelée «publique " qui régit les rapports au religieux en Belgique.

\section{Conclusion}

Le pentecôtisme, tel que pratiqué à Bruxelles, est investi de manière intense dans un contexte de migration. Deux temporalités se dessinent dans les trajectoires migratoires et il semblerait que ces Églises interviennent distinctement lors de ces différentes étapes. Premièrement, et dans une certaine mesure, le facteur religieux semble pallier le manque de clarté et d'encadrement de l'État belge dans la gestion des personnes en situation irrégulière sur le territoire. Le système produit des "exclus de l'intérieur qui sont d'une certaine manière tolérés " bien qu'ils vivent en dehors de toute reconnaissance des droits sociaux fondamentaux (Adam, Ben Mohammed, Kagné, Martiniello, Réa, 2002 : 210). Le ralliement à une église et à une "communauté de destin " (Sayad, 1993 : 1269) peut être compris, dans ce contexte précis, comme une tentative de ces " exclus de l'intérieur » de s'insérer dans un espace réconfortant mais aussi de retrouver un sentiment d'appartenance jusqu'alors fragilisé par l'expérience migratoire. Le langage pentecôtiste est l'occasion d'une mise en récit alternative de cette expérience. Dans ce récit, les migrants ne sont pas réduits à n'être que des individus en quête d'une plus grande stabilité politique ou des profiteurs du système économique, mais ils deviennent, à l'instar du peuple d'Israël, un peuple choisi par Dieu pour faire « de grandes choses » dans leur pays d'accueil. Les fidèles voient la «main de Dieu » à l'œuvre à différents niveaux du processus d'installation et "d'intégration» dans le pays d'accueil. Leur présence sur ce territoire est comprise comme le résultat d'une volonté divine. Qu'il s'agisse de l'obtention d'un visa pour toute la famille ou de la «voix de Dieu » qui retentit lors d'un premier voyage en Europe, nombreux sont les récits qui font intervenir directement le plan divin dans le choix du pays de destination.

Le second temps migratoire est celui de la désillusion des fidèles plus anciennement établis. Le discours s'y référant n'est plus celui de l'intégration et de l'assimilation mais celui d'une mise en perspective de la différence; qu'il soit 
question de l'irréductibilité de leur différence ou de la différence en creux sur le plan des «valeurs spirituelles » associées aux Belges. D’abord, les fidèles constatent que malgré leurs efforts pour ressembler aux non immigrés, ils continuent d'être considérés comme "différents » y compris ceux qui sont nés en Belgique et qui ont grandi selon un mode vie « à la belge ». Les migrants, ou leurs descendants, au travers de la mobilisation du discours religieux et de ses multiples déclinaisons, peuvent alors énoncer ce qui est difficilement énonçable par ailleurs : la situation humiliante de l'attente, le rejet et l'exclusion qu'ils ressentent, l'effondrement du mythe de l'Europe/Eldorado qui les avait bien souvent guidés jusque là. Ce discours donne du sens et répond à ces sentiments confus. Par la suite, on ne veut plus ressembler à ces Belges (surtout au prix de la soi-disant « intégration ») qui sont associés au « monde du mal ». Leur différence est diabolisée et cristallisée autour de l'idée d'immoralité. Cette diabolisation apparaît comme une tentative de remise à niveau ou parfois de renversement de l'asymétrie des rapports de force. Le groupe se présente comme le sauveur d'une société européenne en perdition et acquiert, ainsi, un rôle utile dans la société d'accueil. Les fidèles pentecôtistes réagissent aux catégories d'attribution par lesquelles ils sont identifiés de manière négative pour retracer leur propre auto-genèse où, on l'a vu, Dieu a un rôle important. Ils seraient, à les en croire, les acteurs futurs d'un processus de moralisation et de réorganisation de la société. En ce sens, le facteur religieux constitue un levier dynamique d'appréhension de la réalité sociale et donne un ancrage significatif à l'inscription du groupe dans un nouvel espace national.

Cependant, si cette moralisation est l'objet d'un discours, elle n'a pas d'incidence réelle sur les Belges non immigrés de la société d'accueil qui accueillent avec indifférence ce jugement. On peut d'ailleurs se demander si l'objectif est réellement la transformation de la société ou s'il n'est pas plutôt la présentation et l'actualisation d'une image valorisante du groupe. L'argument disqualifiant les modes de vie des Européens viendrait alors répondre à celui qui stigmatise les « outsiders » pour cause d'illégitimité sur un territoire qu'ils n'ont pas contribué à «constituer ». Il s'agit pour les populations discriminées de se construire une identité positive et de se réapproprier, dans un contexte spécifique, leur légitimité perdue. Ces processus de dévalorisation et de revalorisation en cascade sont le résultat d'un retournement assez classique de la structure de mépris et soulignent l'importance du caractère dialectique dans la construction des frontières culturelles. Les oscillations, tensions et contradictions du discours des Églises pentecôtistes attestent finalement de l'ambiguïté des liens entretenus avec les nationaux, entre fascination et diabolisation.

Maïté MASKENS

Bruxelles, Centre Interdisciplinaire d'Étude des Religions et de la Laïcité

mmaskens@ulb.ac.be 


\section{Bibliographie}

Adam Ilke, Ben Mohammed Nadia, Kagné Bonaventure, Martiniello Marco, Réa Andréa, 2002, Histoires sans-papiers, Bruxelles, Éditions Vistas.

BARTH Frederic, 1969, Ethnic Groups and Boundaries. The Social Organization of Culture Difference, Oslo, Universitetsforlaget.

BASTENIER Albert, 1998, "L'incidence du facteur religieux dans la "conscience ethnique" des immigrés marocains en Belgique ", Social Compass, 45-2, pp. 195-218.

BAva Sophie, 2003, "Les Cheikhs mourides itinérants et l'espace de la ziyâra à Marseille ", Anthropologie et Sociétés, 27-1, pp. 149-166.

BANCEL Nicolas, BlanCHARd Pascal, 1997, « De l'indigène à l'immigré : images, messages et réalités ", Hommes et Migrations, 1207, pp. 6-32.

BlANES Ruy Llera, 2006, "The atheist anthropologist: Believers and non-believers in anthropological fieldwork ", Social Anthropology, 14-2, pp. 223-234.

BRUBACKer Rogers, COOPER Frederick, 2000, "Beyond "Identity ", Theory and Society, 29-1, pp. 1-47.

CoRnet Anne, 2004, "Les Congolais en Belgique aux XIX et Xx ${ }^{\mathrm{e}}$ siècles ", in Morelli A., (dir.), Histoire des étrangers et de l'immigration en Belgique de la préhistoire à nos jours, Bruxelles, Couleurs Livres, pp. 375-400.

CORTEN André, MARY André, 2001, "Introduction ", in Corten A., Mary A., (éds.), Imaginaires politiques et pentecôtismes: Afrique/Amérique Latine, Paris, Karthala, pp. 11-33.

Corten André, Marshall-Fratani Ruth, 2001, (éds.), Between Babel and Pentecost. Transnational Pentecostalism in Africa and Latin America, Bloomington, Indiana University Press.

Corten André, Dozon Jean-Pierre, Oro Ari Pedro, 2003, "Introduction », in Corten A., Dozon J.-P., Oro A.-P., (éds.), Les nouveaux conquérants de la foi. L'Église universelle du royaume de Dieu (Brésil), Paris, Karthala, pp. 13-35.

CORTEN André, «Le renouveau religieux pentecôtiste. Éléments d'explication politicothéologique ", PentecoStudies, 3-2, http://www.glopent.net/pentecostudies/2004/ corten2004.pdf/view, consulté le 26 décembre 2007.

DANDOY Michel, 2005, Le protestantisme: mémoires et perspectives, Bruxelles, Éditions Racines.

Devisch René, 1998, "La violence à Kinshasa, ou l'institution en négatif ", Cahiers d'études africaines, 150/152, pp. 441-469.

-, 2001, "Les Églises de guérison à Kinshasa. Leur domestication de la crise des institutions ", in Corten A., Mary A., (éds.), Imaginaires politiques et pentecôtismes : Afrique/Amérique Latine, Paris, Karthala, pp. 119-141.

Duquesne Antoine, Willems Luc, 1997, Rapport de la Chambre des Représentants de Belgique, "Enquête parlementaire visant à élaborer une politique en vue de lutter contre les pratiques illégales des sectes et le danger qu'elles représentent pour la société et pour les personnes, particulièrement les mineurs d'âge ", Troisième session de la $49^{\mathrm{e}}$ législature, 28 avril 1997, Partie II.

FANCELlo Sandra, 2005, "Pouvoirs et protections des femmes dans les Églises pentecôtistes africaines ", Revista de Estudos da Religião, 3, pp. 78- 98.

-, 2006, Les aventuriers du pentecôtisme ghanéen. Nation, conversion et délivrance en Afrique de l'Ouest, Paris, IRD-Karthala. 
GIFFORD Paul, 2001, «The complex provenance of some elements of African pentecostal theology ", in Corten A., Marshall-Fratani R., (éds.), Between Babel and Pentecost. Transnational Pentecostalism in Africa and Latin America, Bloomington, Indiana University Press, pp. 62-79.

Glick Schiller Nina, Çaglar Ayse, Guldbrandsen Thaddeus C., 2006, « Beyond the ethnic lens: Locality, globality, and born again incorporation ", American Ethnologist, 33-4, pp. 612-633.

Hervieu-LÉger Danièle, Davie Grace, 1996, « Le déferlement spirituel des nouveaux mouvements religieux ", in Hervieu-Léger D., Davie G., (dir.), Identités religieuses en Europe, Paris, La Découverte, pp. 269-289.

Kagné Bonaventure, Martiniello Marco, 2001, "L’immigration subsaharienne en Belgique ", Courrier hebdomadaire, 1721, pp. 5-49.

LE GALL Josiane, 2003, "Le rapport à l'islam des musulmanes shi'ites libanaises à Montréal ", Anthropologie et Sociétés, 27-1, pp. 131-148.

LuCA Nathalie, 2007, "La conversion comme conflit de citoyenneté ", Communication au colloque de l'AFSR, Paris, 5-6 février 2007.

Martiniello Marco, 2001, La nouvelle Europe migratoire. Pour une politique proactive de l'immigration, Bruxelles, Éditions Labor.

MeInTEl Deirdre, Leblanc Marie-Nathalie, 2003, «La mobilité du religieux à l'ère de la globalisation ", Anthropologie et Sociétés, 27-1, pp. 5-11.

Misenga Monique, 2000, "Diasporas africaines : entre indépendantisme et intégration. Analyse de trois groupes religieux africains en Wallonie et à Bruxelles ", in Spindler M., Bart-Lenoble A., (dir.), Chrétiens d'outre-mer en Europe. Un autre visage de l'immigration, Paris, Karthala, pp. 239-248.

Maskens Maïté, Noret Joël, 2007, « La Nouvelle Jérusalem. Éléments d'histoire et de sociologie d'une Église pentecôtiste en Belgique », Le Figuier, 1, pp. 117-137.

Mossière Géraldine, 2007, "Sharing in ritual effervescence: Emotions and empathy in fieldwork ", Anthropology Matters Journal, 9-1, http://www.anthropologymatters .com/journal/2007-1/mossiere_2007_sharing.htm, consulté le 2 mai 2007.

Noret Joël, 2005, "Logiques transnationales et développements missionnaires dans les pentecôtismes nigérian, béninois et togolais ", in Fourchard L., Mary A., Otayek R., (éds.), Entreprises religieuses transnationales en Afrique de l'Ouest, Paris, IFRAKarthala, pp. 417-438.

PÉDron-Colombani Sylvie, 1998, Le pentecôtisme au Guatemala : conversion et identité, Paris, CNRS Éditions.

RÉA Andréa, 1998, «La culture comme résistance, identités et stratégies politiques », in Bouras D., (coord.), Immigration, diversité culturelle et démocratie, Actes de la table ronde du 24 avril 1998, Bruxelles, Éditions Luc Pire et le PAC, pp. 82- 89.

RÉA Andréa, Tripier Maryse, 2003, Sociologie de la migration, Paris, La Découverte.

Robisns Joel, 2004, "The globalization of pentecostal and charismatic christianity ", Annual Revue of Anthropology, 33, pp. 117-143.

SAYAD Abdelmalek, 1993, "La malédiction », in Bourdieu P. (dir.), La misère du monde, Paris, Seuil, pp. 1267-1300.

SPINDLER Marc, 2000, «L'implantation d'Églises d'outre-mer en Europe : aspects missiologiques ", in Spindler M., Bart-Lenoble A., (dir.), Chrétiens d'outre-mer en Europe. Un autre visage de l'immigration, Paris, Karthala, pp. 21-35. 
Ter HaAr Gerrie, 1998, Halfway to Paradise: African Christians in Europe, Londres, Cardiff Academic Press.

UGba Abel, 2006, "African pentecostals in $21^{\text {st }}$ century Ireland ", Studies, 95/378, pp. 163-173.

Willaime Jean-Paul, 1992, La précarité protestante. Sociologie du protestantisme contemporain, Genève, Labor et Fides.

-, 1999, "Le pentecôtisme : contours et paradoxes d'un protestantisme émotionnel ", Archives des Sciences Sociales des Religions, 105, pp. 5-28.

\section{Résumé}

Des Églises pentecôtistes portées par une majorité de fidèles originaires d'Afrique subsaharienne et d'Amérique Latine se multiplient à Bruxelles depuis une trentaine d'années. La problématique de la migration a une place importante dans le discours et les pratiques de ces nouveaux venus. Qu'il s'agisse de la figure d'un "Dieu des réfugiés" ou de pratiques d'entraide au sein de l'Église, le contexte de migration colore leur foi de manière particulière. Cet article montre que le facteur religieux donne du sens à leur présence dans le pays d'accueil. La vie religieuse quotidienne constitue un espace positif d'auto-identification du groupe de fidèles en réponse à la discrimination dont ils sont généralement victimes en tant que minorités immigrées. L'adhésion au pentecôtisme est un point d'appui aux migrants et, dans le même mouvement, le discours religieux contribue à construire l'expérience migratoire.

Mots-clés: Minorités immigrées, Églises pentecôtistes, discrimination, intégration, identité chrétienne.

\section{Abstract}

During the past thirty years, Pentecostal churches, mostly composed by followers from Subsaharian Africa and Latin America, blossomed in Brussels. In this article, I intend to show that migration issues are a crucial dimension of these newcomers' way of talking about and practicing their religion. As I will demonstrate, they influence faith in a particular manner. Under the protection of a "God of the refugees" and benefiting from mutual social support within the Church, migrants develop a specific religious lifestyle that contributes to give meaning to their contested presence in the host country. In particular, the Church constitutes a positive self-identification space for the followers who, as immigrants, are often discriminated from the rest of the population. In a nutshell, this paper looks at how Pentecostal religious movements and discourses support and give meaning to migrants' experiences.

Key words: Immigrants minorities, Pentecostal churches, discrimination, integration, christian identity.

\section{Resumen}

Las iglesias pentecostales, traídas por una mayoría de fieles originarios del África (central y meridional) y de América Latina, aparecen en Bruselas desde hace treinta años. La problemática de la migración tiene así un sitio importante en el discurso y las prácticas de estos recién llegados. Se trata de la figura de un "Dios de los refugiados" o de actividades de ayuda mutua en el seno de la iglesia. El contexto de la 
68 - Archives de sciences sociales des Religions

migración colorea la fe de manera particular. El factor religioso da sentido a su presencia en el país receptor. La vida religiosa diaria constituye un espacio positivo de auto-identificación del grupo de fieles en respuesta a la discriminación de la que generalmente son víctimas como minorías inmigradas. En este marco, la adhesión a la religión es un soporte en el inmigrante, y al mismo tiempo, el discurso pentecostal contribuye a construir la experiencia migratoria.

Palabras clave: Minorías inmigrantes, iglesias pentecostales, discriminación, integración, identidad cristiana. 